https://doi.org/10.46344/JBINO.2021.v10i02.31

\title{
PROTEIN-PROFEN INTERACTION: THE ROLE OF CARBON IN DEALING ACTIVE SITE INTERACTION
}

\author{
Rajasekaran Ekambaram
}

IV.S.B. Engineering College, Karur-639111, Tamil Nadu, India.

\section{Email: ersekaran@gmail.com}

\begin{abstract}
Nano force coming from carbon alone was part of our discussions in the earlier calculations. It is found out to be the predominant role of carbon coming from adequacy principle governed from carbon value. Here it is extended to protein-ibuprofen interaction as case study for demonstrating the role played by value of carbon in dealing with internal healing of newly developed situation arising from carbon value. Well all of us sudden take place that the internal carbon demonstrated to be in par with cohesiveness arising out of healing path of drug action. Very well done with interaction and associated movement of atoms in dealing cohesiveness in the protein to accommodate incoming drug of action. Whereas it is noticed that the internal carbon optimized domain (COD) adjusted to be the main part of the dealing with drug delivery to active site rather than van der Waals and electrostatic forces of interaction. Only those that are in accordance are adjusted to new development and all that are rigid are unaltered during intercourse of action. It is noticed that the interaction arising from carbon role will be the main part of interaction that needed to be measured from bond of all atoms involved. In this context, whether or not the measurable quantity may be deviation of the position of individual atoms before and after. We have tested that too and noticed the deviation of internal COD forming elements. That is discussed as part of this new formation of cohesive force and adjustments arising from drug alone are interpreted. With this advancement we might even think of alternative elements to be considered for healing and all. It is going to be the new beginning of alternative treatment for the suffering coming from carbon role and all.
\end{abstract}

Keywords: Ibuprofen; nano; nanotechnology; nanobiology; active site; drug-protein interaction; nanoforce; COD; GAD; 


\section{Introduction}

\section{Cohesiveness of Proteins}

Nano force comes into action in the proteins that can alter or offer course of interaction by combining into another macromolecule. Accordingly the nano device selects to perform the interactive interaction in terms of nano carbon performance in the vicinity of interactive elements. According to nano carbon performance the selected elements are involved with interaction in forming cohesive force of interaction in terms of others also involved in it and into action of force by forming active elements of interaction [Indupriya et al. 2019a, b, Rajasekaran et al 2018, 2019a, b, c, d, e, 2020a, b, Pramanik et al. 2020]. Overall these elements are in force provided carbon value of $31.44 \%$. Otherwise these elements are not in force pushing the situation to inadequate system of interactive elements with neighboring one. To meet out the inadequacy of carbon atoms one might want to mutate and accommodate another for neutralization. Whereas it is possible only those amino acids which are in accordance with carbon value as neighbor needed to be finding out with other carbon coded one performing new situation. While only those that are in accordance can be mutated for satisfying carbon of all situations, the essence of all other interaction elements changed by that may not be known. Otherwise call it as cohesive one coming from neighboring amino acids. One should think of these set of amino acids that can be part of the newly formed profound nature of interactive elements where no other can interfere with any other elements coming from neighboring one. Over and above one should arrange to provide these set of amino acids for the forth coming selection of mutational studies. Well we have it one from our CARd program that takes all is protein sequence and nothing else. Only then it performs some statistical movement of elemental calculation for feasible identity and acceptance of that can perform better for the situation of interest. Over and above this program indentifies all in one go for mutational value of acceptance and also negligence parameter for the avoidable situation. Whereas it is easy to tell that with in the short span of sequence the mutation is acceptable or not and also predictive avoidance score of negligence. Over and above only those elements of interactive amino acids are in accordance with carbon value. Whereas it is of interest for the particular situation where the amino acids is in danger of alteration.

\section{Role of Drug in Dealing Probe-Protein Interaction}

Drug-protein interaction ofcourse be the main part of this study where incoming probe will be binding to newly formed situation or not. Only those situations that can alter arranged amino acids for meaningful accommodation of that probe from being nothing to COD of all atom interaction. Only those arranged amino acids can perform alteration at all level of accommodation may be accepted. Very well done in this regard that any situation 
might be interactive [Rajasekaran 2019b]. But the internal one being bound by new situation will stay for time being while the other may not in the formation at all which again may be going ahead with other kneeling force of interaction coming from all sort of other molecular interaction which may be adjacent to the intervening elements. Only those that are in accordance may be forming meaningful formation of interactive complex forming intercourse of elemental salvation. New one may accepted provided it is satisfying the carbon of all atom according to rule of law coming from carbon alone. When in need one might be interested in leaving the situation to carbon value of role coming into action. Whereas it is going to be wastage of time and limit in performing all atom based calculations coming from force field of interaction coming out of van der Waals, electrostatic or non bonded interactive force while leave out the situation for bad rather than solution. Only those that are in accordance with carbon value will be the meaningful calculations in the years to come. Well it is already in part of our value prediction in our lab for any eventuality arising from carbon value whereas wet lab prediction might be of interest of our eventuality that cause new situation where disease monitor and alteration for remedial course of action that may have to be done fast. Over and above the remedial may be permanent for the situation arising out of certain diseases. When in need one can go on altering new situation permanently rather than hanging around with meal of interest with existing disease monitor. According to new situation one may think of total eradication and new life for betterment and all. Except that it may arise out of situation to situation when in need. Whereas it going to be new exercise in the years to come with all adequacy principle coming from carbon value alone. Within this frame work one can even think of twice the newly one formed out of the attractive elements coming from role of carbon. Whereas it is new thinking in the direction of carbon force of attractive elements arising out of the development from here. In this situation where all other may not work it is possible for one to get cured out of all that suffering. Wait the situation that are amenable for new development and all.

\section{Current work}

Here we continued with carbon analysis of drug of action in dealing with thorough understanding of alteration accordingly. Exactly it is understood to have the carbon role of healing in ibuprofen interaction with protein of interest. Well one might be interested to know how the healing of pain that accordance to carbon value is evaluated. Very well known lipid binding protein (LBP) interaction to ibuprofen is case study here in this calculation of carbon value. Whereas it is demonstrated to be the interaction coming from carbon role where healing take place which tells that even newly formed one could have the same situation form any such healing and all. With this advancement the new carbon role of action will be created for 
series of action for those that can perform well for the newly developing situation. Over and above the development action would be according to the nature of interaction arising out of carbon value here in this study.

\section{Materials and Methods}

\section{CARd Verified Value}

The crystal structures and sequences of human lipid-binding protein (LBP), apo form (3rzy) and in complex with (R) ibuprofen (3p6g) are taken all for calculation. According to requirement the X-Ray structure must be added with hydrogen atoms isolating unwanted stuff from pdb one. Only then it is found for calculation regarding internal COD (ICOD) of all living elements inside the protein for verification of internal COD and all other regarding lively stuff of intercourse material that may or may not happen. Only those elements are found for suitable in the calculation included and neglected the other formality incoming atoms and all. Only the residual considerations are accommodated for any such calculation involving ICOD calculation and superposition validation. Only those that of atoms in the vicinity of undergoing interaction are considered for calculations. Otherwise there must be associated action arising out of intercourse in the next level of operation. Over and above it is one of the tedious jobs of finding it. Whereas it may be possible based on already existing database of other system interaction exist in our nature that can be incorporated for a better tomorrow of disease free world of operation. Only the incoming going to be operational at the level of atom over and above nano of operation. Only those elements are considered for calculations are within dia of $16 \AA$ and may be more and less.

Carbon adaptation in protein 3D structure is governed by the aminoacid in the sequence (Globular Amphipathic DomainGAD). Then the adaptive force coming from amino acids must be spelt out at aminoacid chain. This is captured very well by our CARd sequence analyser. According to neutralisation process of amino acids for global stabilization, those that are flexible side chains are accommodated very easily. Whereas other flat are considered to be more carbon and non flexible one, forming nonICOD regions. It is captured to be active form of work here in protein to be active all along the globular protein.

\section{CARd of 3D Structure Analysis}

All along the analysis it is found out that 3D structure of protein must possess $31.44 \%$ of carbon for its stable structure locally and all globally. It is found out that all are in terms with adequacy principle. At the same time the active site may have a different role of carbon value for its activity to be accordingly. Additional role is played here in active one. According to all our calculations, all active sites are accumulation of carbon and order in different role. Otherwise it is consistent with nonCOD of active protein. Only the adjacent roles are satisfied here as internal COD which is playing in neutralizing the 
carbon adequacy role played for all along the native protein. One or more amino acids are responsible for this kind of attraction adjacent to neutral role. All protein internals are either COD or nonCOD. Only those are not in line of COD tend to form the neutralizing role using incoming role by nullifying game played by Ibuprofen which are captured easily by CARd of all atom calculation adopted in computer programming. Only those are in tune to neutralizing points are very well captured. According to rule of law there must be adequate carbon internally to set neutral the adequacy principle. Otherwise there are neutral carbon satisfying the everywhere role. Only those that are in $16 \AA$ are in principle are considered as a fact of arrangements according to rule of law governed from carbon fact of all atom adoptive principle which are essential here to do away with any part of interaction. Rest van der Waals or electrostatic force may not be important to be considered for interactive role from drug for neutralization of all atoms considered to be a major role. Only those elements involved in neutralization may be captured from superposition values adopted here in this work. Only here the values are reported otherwise mentioned elsewhere in the previous calculation of other work related to carbon value profile pack.

\section{Superposition Point of Valuation}

Well it is understood to be a carbon role adjusted everywhere in the universal of protein to be considered, only those elements that are flexible enough to accommodate the changes are considered as adjustment parameter which again the ICOD valued amino acids and all. Only those ICOD parameter one are adjusted all along the protein to incorporate the drug to binding in the site of interest. According to rule law the governing body of carbon value are adjusted according the rule of law adjust to the neutral one. Only those elements adjacent to active point are adjusted clearly visible from superposition values. Whereas the incoming molecule of action may merely adjust those parameters accordingly and alteration felt at the site along the packing is done of all where adjacent atoms adjusted its position from the original site of native form. Clearly it is evidence and identification of active form of protein in the vicinity of protein pack for its role of activity whereas only those of atoms along the protein may modified for better and adopt new nonCOD site where another may bind rerun the whole thing of fundamental principle of protein in action. Only those active roles are captured as unaltered during superposition. Rest all adjusted to it. Whereas in need of alteration at the site where active point is located the chemistry of altered role may be interesting to note while other position may not form accordingly. Very many neutralization points are captured where there may not have any active site of interest where adjacent one role is to be monitored for action in this case of adjustment. According to role played by drug molecule may be limited to some changes but all along the changes in felt 
accordingly. Only those are captured may be affected by this action of all atom force coming from drug alone. Only those changes may influence many other actions to be followed where every atom is involved with every other atom at the juncture at dia $16 \AA$. Bond of all atoms in the vicinity of ICOD found out to be small when compared to one in another mode. Only those are in form of ICOD adjusted according to nature of interaction internally to the external one. All these changes are accommodated in the displacement when drug bind to active site. Every then there, there might be change in ICOD value that can improve or become nonCOD. Only those that are nonCOD are considered for further course of action for any other interaction in the universe protein action the bottom of line. Every now and then the same procedure is repeated for all along the work of interest when in need. Whereas other amino acid portion may have active role. Only those that are in rich of carbon may take part in the due course of action. Well only those are in rich carbon portion must be eligible for interactive role to be done in the interface of incoming molecule. Whereas all other portion of interest might be simply attractive and may not involved with intercourse of action with neighbor one. When in need of such action one would think of introducing active aminoacid here in the part for performance based action. When any molecule to be binding to newly earned active site, other might be enrolling for its neutralization activity. Elsewhere the activity take place means it found to be inactive with intervening form of action in the course of action taken during intercourse of one and only molecule to bind. Over and above it is decided by intervening amino acids and all. All that happens because of ICOD changes adjacent to active form of amino acids. Only then it is accommodated here as active form elsewhere in the system interest. Role of carbon is key to be the interest in all these intercourse of action. Any deviation from this intervening action may be because of nonCOD of all atoms involved in it. Whereas it is found out to be inaction in ICOD one. When one required to be active, all they have to do is introduce amino acids that are flat and carbon rich. Otherwise going to be ICOD one with elements of non carbon one and all. Whereas it is recommended that all those that are flat may be inconsistent with ICOD formation which need to eliminated for any such switching off function. When one wants test this situation, please contact here as our laboratory focused on it. Very well done with all other system of known examples and explained the situation. Whereas it is recommended that any change in amino acid sequence with deliberate attempt to change property or function might be loss of effort and interest. Only carbon decides to be in charge of all these happening and needed to be focused. Any change in need, please direct to the email above and ask for particular change. Otherwise all other kind of mutational study might not yield result accordingly. Ask for any demo for such interactive role of carbon based intuition 
coming from aminoacid of altered one. Very well documented everywhere in the system of universe where it is going to be dominant in the due course of action.

\section{Results and Discussion}

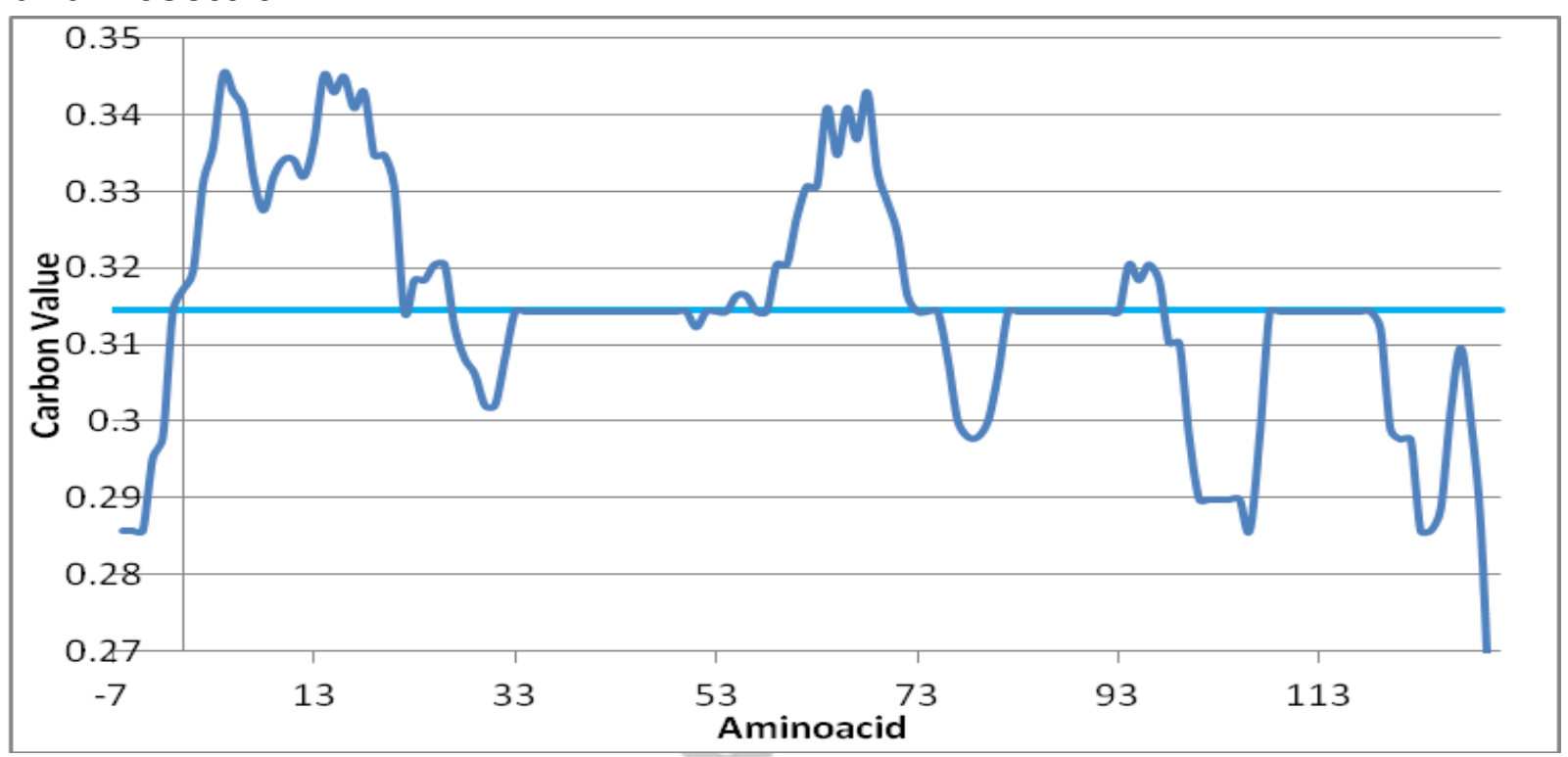

Figure 1: GAD of all amino acids along the sequence is shown. Active site (59-72) is clearly visible from carbon portion whereas ICOD (34-57) portion are along the line of ICOD value 0.3144 . Sometime 73-93 is considered as ICOD region even though there are some hydrophilic elements. Also noteworthy that the carbon high portion of $0-23$ and hydrophilic portion of $97-108$ and 118-131 are visible from the CARd program of interest.

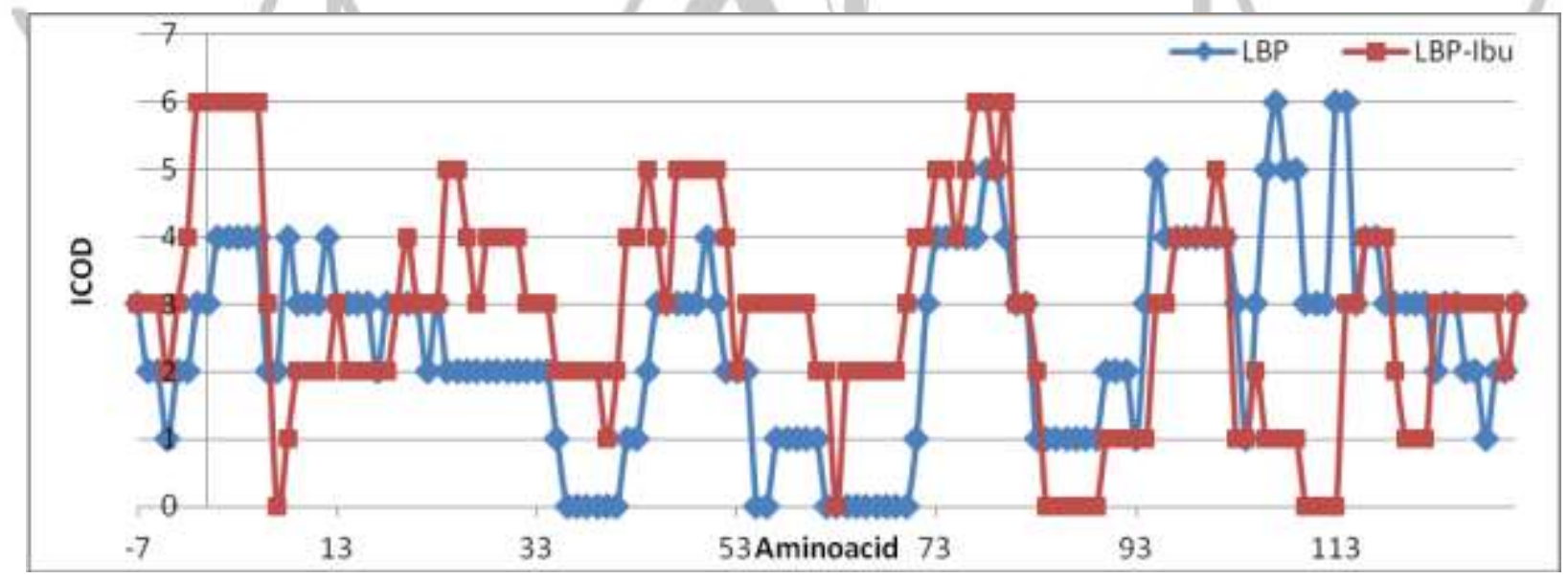

Figure 2: ICOD versus aminoacid plot using 3D structure of LBP (blue) and in complex with Ibuprofen (dark brown). Note the active site (62-70) nonICOD becomes ICOD. Neighboring ICOD (41-61 and 70-83) remain same both in LBP and complex one. 


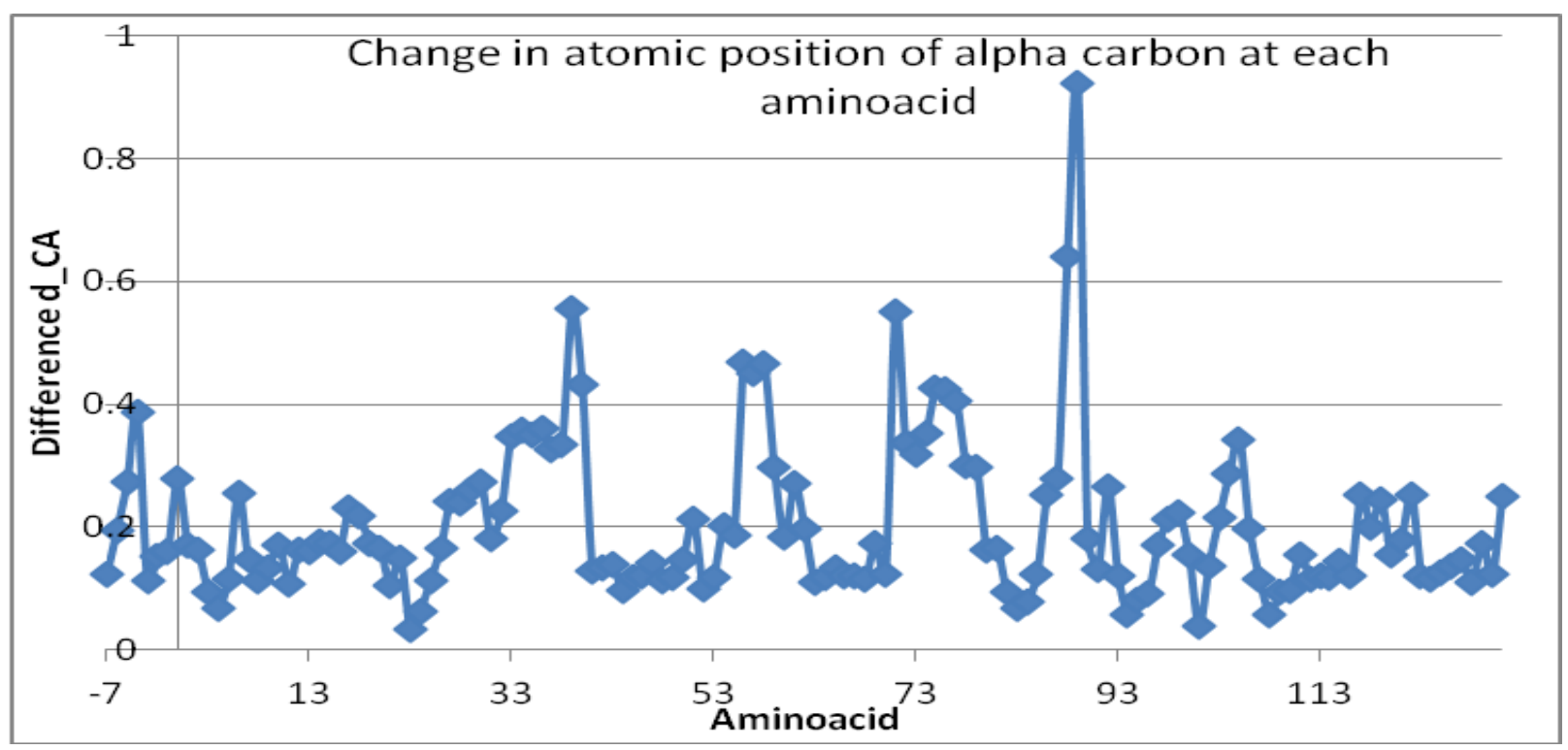

Figure 3: Alteration of position of alpha carbon (CA) at each aminoacid. Note that there is no change in active site (63-70) and maximum at adjacent domain regions (53-62 and 7181).

It is clear that the binding site (63-70) is not all affected from binding to drug one. Only the internal COD amino acids get adjusted for the need of binding and application to other matter of intercourse between incoming probe for fruitful results of relief. Way back in intuition the internal COD altered accordingly and adjust for satisfying the new situation for stable adequacy principle coming from carbon rule of law that alter other portion as well. Over and above only flexible amino acids are involved in these kind of alteration and need fulfillment at active site whereas the other flat amino acids may not follow the internal COD stuff and others to follow in principle while working for certain intercourse action. Only then the principle is met for the action to be taken and allow the others to accommodate need based adjustment and all.
Another program to find the unusual aminoacid templates is finding that 2-8, 1320 and 61-72 are carbon rich regions. Whereas the another expected template of aminoacid consisting of $C, F, P, W, Y$ are not here in this case whereas it is part of the active site most of the time somewhere else. Also the carbon high elements are found to be balanced by carbonless stretches at 76-82 and 100-108. 60TEISFILGQEF70 is the active site stretch that contain phenylalanine at $64^{\text {th }}$ position is the crucial one for activity of all that happen everywhere in the universe of binding capability during painful action in the internal matter that can accommodate all of one may be crucial when compared to any other force of interaction within the cell function for pain relief over and above the reality show for anything that can take one at that time of 
work for painful exchange between nature and all.

\section{Conclusion}

Protein-Probe interaction at nano level is revealed here in terms of healing process that can accommodate all sufferings arising out of carbon alone. Only those that are accordance with carbon value are explained here in this case study report. Whereas it is expected to have many fold of action in the years to come. Healing the painful action under control is part of the nature of intuition in the cell function where all other forces fail to explain. Only the interaction coming from carbon value is demonstrated to be the healing all processes that come into coexistence of life and matter where healing is part of it. Well we have demonstrated that in this case of interaction of carbon role adequately during interactive elements into play at the site of active role and all. Only those that are involved in biding are explained here in terms of carbon value rather than one coming from alternative force of interaction coming from van der Waals or electrostatic one. Only time will tell all that take place and adequacy principle needed to be followed for protein probe interaction for different situation arising out of disease monitor.

\section{References}

1. Indupriya $R$, Meenal $R$, Kavitha $V$, Rajasekaran E. 2019a. Drug-protein interaction validates the internal COD formed due to cohesive force: Test of bond length variation in amino acids involved. Int J Mol Biol Open Access. 4: 113-117.

2. Indupriya R, Meenal R, Rajasekaran E. 2019b. Existence of carbon domain alters bond orders in protein. Int J Inno Eng Tech. 13: 128-132.

3. Rajasekaran E. 2018. Domains based in carbon dictate here the possible arrangement of all chemistry for biology. Int. J. Mol Biol-Open Access. 3: 240-243.

4. Pramanik $S$, Thaker $M$, Perumal AG, Rajasekaran E, Poondla N, Schmidt M, Kim PS, Kutzner A, Heese K. 2020. Proteomic atomics reveals a distinctive uracil-5methyltransferase. Molecular informatics. 39:1900135.

5. Rajasekaran E, Meenal R, Michael PA, Indupriya R. 2019a. Existence of nano level force in protein plays applications of maximum untold understanding of life form. Int J Eng Adv Tech. 9: 3722-3726.

6. Rajasekaran E, Indupriya R, Meenal R. 2019b. Domain formation in regions of protein probe interaction. Int $\mathrm{J}$ Mol Biol Open Access. 4:167-169.

7. Rajasekaran E, Kavitha V, Ganeshbabu P, Prabakaran R, Meenal R, Indupriya R. 2019c. Nature of amino acid sequence instruct carbon value to be adopted in protein 3D structure. IEEE Access. 10541060.

8. Rajasekaran E, Indupriya R. 2019d. Who power sickle cell disease: Carbon domain analysis tells all because of design in protein 3D arbitrary internal carbon 
domain (COD) arrangement, Int J Mol Biol Open Access. 4:85-88.

9. Rajasekaran E, Meenal R, Indupriya R, Prabakaran R, Boobalan S, Jayato N, Sivakumar K, Kalaivani T, Rathika GM, Saranya K, Brindha G. 2019e. Existence of cohesive force explains all phenomena that are in material which holds strong bond of all forces of attraction: A case study with carbon material. AIP Conference Proceedings. 2087:020015.
10. Rajasekaran E, Meenal R, Indupriya R. 2020a. Study on aquaporin proves to be the carbon in protein-protein interface playing in tetramerisation. High Tech Lett. 26(5):292-298.

11. Rajasekaran E, Meenal R, Indupriya R. 2020b. Carbon role in the form of action of intelligence in the living being. J Study Res. 12:66-72.
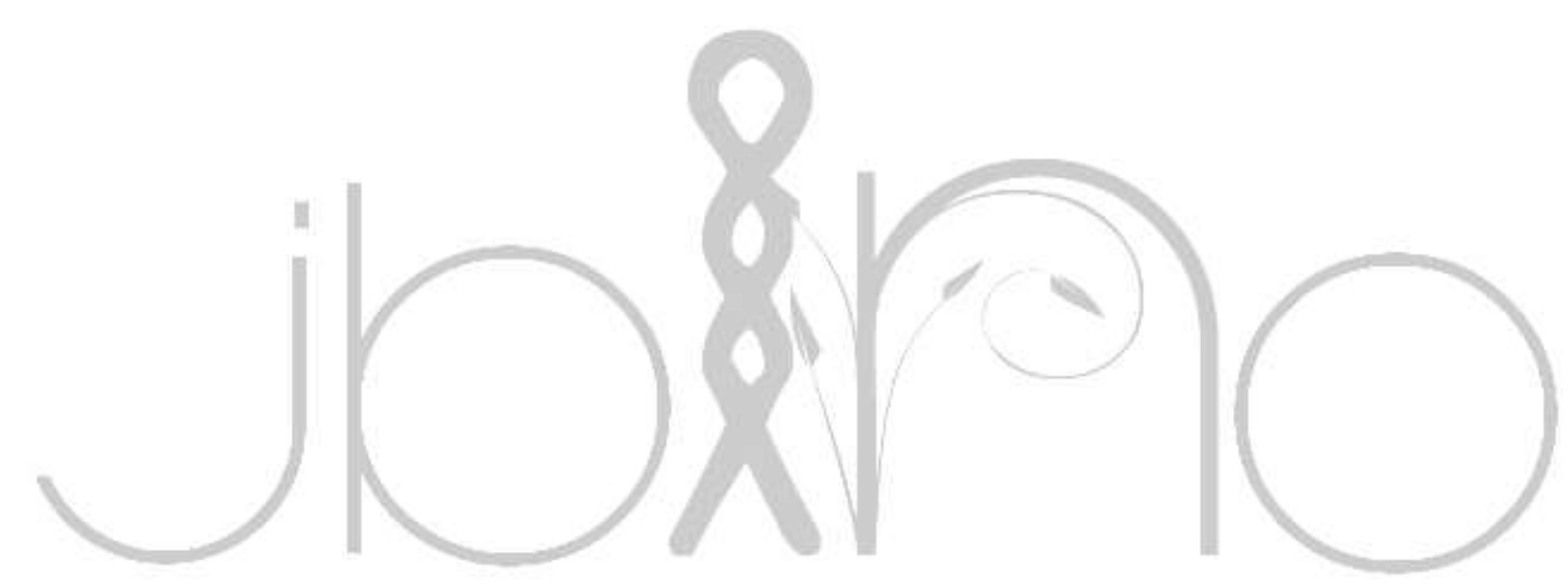